\title{
Short Call Author Abstract Index
}

Abuchaibe, C. Short Call Poster 29

Afkhami, M. Short Call Poster 71

Albejante, M. Short Call Poster 59

Aldawish, M. Short Call Poster 20

Alghamdi, A. Short Call Poster 20

Alhashemi, A. Short Call Poster 36

Al Kahtany, N. Short Call Poster 20

Al-Nuaim, A. Short Call Poster 47

Alsayed, M. Short Call Poster 10

Antonelli, A. Short Call Poster 14, Short Call

Poster 15

Arata, N. Short Call Oral 5

Ashkenazi, K. Short Call Poster 52

Astapova, I. Short Call Poster 27

Bach, A. Short Call Oral 7

Bagheri-Yarmand, R. Short Call Oral 3

Baker, A. Short Call Poster 17

Balázs, G. Short Call Poster 53

Bar, D. Short Call Poster 52

Bardet, S. Short Call Oral 6

Barnes, J.W. Short Call Poster 13

Bastholt, L. Short Call Poster 42

Batista, S.J. Short Call Poster 39

Beadnell, T.C. Short Call Poster 19

Benesch, M. Short Call Oral 1

Benisvy, D. Short Call Oral 6

Berger, M. Short Call Oral 7

Bernard, J. Short Call Poster 42

Bernet, V. Short Call Poster 44

Betances, E.M. Short Call Poster 39

Bianco, A. Short Call Poster 53

Bibb, J. Short Call Poster 24

Biricotti, M. Short Call Poster 14, Short Call Poster 15

Bodnar, N.M. Short Call Poster 12

Böhme, K. Short Call Poster 69

Borget, I. Short Call Oral 6

Borrelli, N. Short Call Poster 64

Boucai, L. Short Call Oral 7, Short Call Poster 40

Bournaud, C. Short Call Oral 6

Bovenzi, C. Short Call Poster 55

Braeth, S. Short Call Poster 31

Brandt, S. Short Call Oral 8

Braverman, L. Short Call Poster 30

Brindley, D. Short Call Oral 1

Brose, M.S. Short Call Poster 42

Budke, B. Short Call Poster 12

Bushman, N. Short Call Poster 68

Caldwell, K.L. Short Call Poster 16 Camacho, C.P. Short Call Poster 59

Carlé, A. Short Call Oral 4
Carpenter, D.J. Short Call Poster 55 Carter, A. Short Call Poster 24

Catargi, B. Short Call Oral 6

Centeno, B.A. Short Call Poster 29

Cerda, S.R. Short Call Poster 30

Charitou, M. Short Call Poster 28

Chen, A.Y. Short Call Oral 8

Chen, R. Short Call Poster 57

Chen, S. Short Call Poster 11

Chen, Z. Short Call Oral 8

Chertman, M. Short Call Poster 63

Chertman, W. Short Call Poster 63

Chindris, A. Short Call Poster 44

Chu, K. Short Call Oral 1

Connor, N. Short Call Poster 68

Coombes, K. Short Call Poster 26

Cottrill, E.E. Short Call Poster 17

Cuellar, M. Short Call Poster 71

Curry, K.M. Short Call Poster 13

da Silva, V. Short Call Poster 59 Daugherty, J. Short Call Poster 12

Daza, E.J. Short Call Poster 23

de Almeida, R. Short Call Poster 59

Deandreis, D. Short Call Oral 6

DelaFuente, M. Short Call Poster 62

Dellê, H. Short Call Poster 59

De Lourdes Fonseca, T. Short Call Poster 53

Dembrowski, G.C. Short Call Poster 13

Dewald, J. Short Call Oral 1

Dias, D. Short Call Poster 21

Dietrich, J.W. Short Call Poster 31

Dip, F. Short Call Poster 62

Doherty, G.M. Short Call Poster 30, Short Call Poster 43

dos Santos Bacigalupo, L.

Short Call Poster 59

Downs, E. Short Call Poster 56

Dromi, N. Short Call Poster 52

Ekpe Adewuyi, E. Short Call Oral 1

Elia, G. Short Call Poster 14, Short Call Poster 15

Elisei, R. Short Call Poster 42

Eszlinger, M. Short Call Poster 69

Faber, J. Short Call Oral 4

Fagin, J. Short Call Oral 7, Short Call Poster 42

Falco, J. Short Call Poster 62

Fallahi, P. Short Call Poster 14, Short Call Poster 15

Fernandes, G.W. Short Call Poster 53

Fernández, S. Short Call Poster 26
Ferrari, S. Short Call Poster 14, Short Call Poster 15

Ferris, R.L. Short Call Oral 8

Finkelstein, S. Short Call Poster 50, Short Call Poster 51

Fish, S. Short Call Oral 7

Forsyth, C. Short Call Poster 53

Frenkiel, S. Short Call Poster 46

$\mathrm{Fu}, \mathrm{G}$. Short Call Oral 2

Fuehrer, D. Short Call Poster 42

Fukata, S. Short Call Oral 5

Gagel, R. Short Call Oral 3

Ganly, I. Short Call Oral 7

Gao, L. Short Call Poster 72

Ghandi Kolli, S. Short Call Poster 50

Ghosh, S. Short Call Oral 1

Godbert, Y. Short Call Oral 6

Godley, F.A. Short Call Poster 30, Short Call Poster 43

Goldenberg, D. Short Call Poster 17

Goldenberg, N. Short Call Poster 12

Gong, F. Short Call Poster 11

Gonzalez, A. Short Call Poster 39

Goodstein, L. Short Call Poster 55

Görke, F. Short Call Poster 69

Grubbs, E.G. Short Call Oral 3

Hache-Marliere, M. Short Call Poster 39

Hanna, A. Short Call Poster 36

Harumy de Lima Hirata, A. Short Call Poster 59

Haugh, K. Short Call Poster 51

Henry, M. Short Call Poster 46

Hidaka, Y. Short Call Oral 5

Hier, M.P. Short Call Poster 46

Hill, C. Short Call Poster 67

Hine, C. Short Call Poster 27

Hollenberg, A.N Short Call Poster 27

Hosono, S. Short Call Poster 50

Hsieh, A. Short Call Oral 2

Hsu, S. Short Call Poster 37

$\mathrm{Hu}$, J. Short Call Poster 23

Inam, S. Short Call Poster 20

Inoue, E. Short Call Oral 5

Isaev, P. Short Call Poster 48

Itani, B. Short Call Poster 20

Ito, S. Short Call Oral 5

Jacquier, J. Short Call Poster 57

Jardine, D. Short Call Poster 67

Jhiang, S. Short Call Poster 26

Ji, Q. Short Call Poster 41 
Jiang, Y. Short Call Poster 11, Short Call Poster 72

Jones, A. Short Call Poster 45

Jones, R.L. Short Call Poster 16

Julie, B. Short Call Oral 8

Kandil, M. Short Call Poster 20

Kelly, A. Short Call Oral 6

Keshavarzian, A. Short Call Poster 53

Khan, S. Short Call Poster 49

Khuri, F.R. Short Call Oral 8

Kim, H. Short Call Poster 61

Kim, J. Short Call Poster 19

Klimczak, D.M. Short Call Poster 19

Klopper, J. Short Call Poster 22

Ko, Y. Short Call Oral 1

Komic, A. Short Call Poster 45

Kosaki, K. Short Call Oral 5

Kosaki, R. Short Call Oral 5

Kruse, E. Short Call Poster 45

Kubota, S. Short Call Oral 5

Kuenzi, B. Short Call Poster 19

Kumar, G. Short Call Poster 50, Short Call Poster 51

Kushchayev, S. Short Call Poster 54

Kushchayeva, Y. Short Call Poster 54

Kushnir, M. Short Call Poster 52

Lai, R. Short Call Oral 1

Lai, X. Short Call Poster 72

Laird, G. Short Call Poster 42

Laurberg, P. Short Call Oral 4

Lawson, A. Short Call Poster 45

Leboulleux, S. Short Call Oral 6, Short Call Poster 42

Lee, M. Short Call Poster 13

Lee, S. Short Call Poster 30, Short Call Poster 43, Short Call Poster 60

Leenhardt, L. Short Call Oral 6

Leite, V. Short Call Poster 21

Li, D. Short Call Oral 7

Li, H. Short Call Poster 66

Li, J. Short Call Poster 11

Liang, J. Short Call Poster 65

Lin, O. Short Call Oral 7

Lin, Y. Short Call Poster 65, Short Call Poster 66

Lithwick Yanai, G. Short Call Poster 52

Liu, R. Short Call Poster 72

Loos, U. Short Call Poster 31

Lopez-Campistrous, A. Short Call Oral 1

Macdonald, C. Short Call Poster 68

Mackay, D. Short Call Poster 60

Mackey, J. Short Call Poster 54

MacMillan, C. Short Call Oral 2

Maghami, E. Short Call Poster 71

Magner, J. Short Call Poster 42

Mahmood, A. Short Call Poster 70

Makhmudov, A. Short Call Poster 16

Manrique, H. Short Call Poster 58

Marques, P. Short Call Poster 21

Martins da Silva, L. Short Call Poster 59

Massoll, N. Short Call Poster 52
Materazzi, G. Short Call Poster 14, Short Call Poster 15

Maxwell, C. Short Call Poster 28

Mcaneny, D. Short Call Poster 30, Short Call Poster 43

McAninch, E. Short Call Poster 53

McFarland, M.A. Short Call Poster 34

McGorisk, G.M. Short Call Oral 8

McIver, B. Short Call Poster 29

McMullen, T. Short Call Oral 1

Meiri, E. Short Call Poster 52

Meltzer, C.J. Short Call Poster 18

Menke, D. Short Call Poster 12

Miao, L. Short Call Poster 61

Miccoli, P. Short Call Poster 14, Short Call Poster 15

Miller, J. Short Call Poster 5

Minkowitz, G. Short Call Oral 7

Miragaya, J. Short Call Poster 34

Mireskandari, A. Short Call Poster 50, Short Call Poster 51

Mishall, K.M. Short Call Poster 19

Mitchel, H. Short Call Poster 52

Mitchell, A. Short Call Poster 67

Mitchell, J. Short Call Poster 27

M L C Bocco, B. Short Call Poster 53

Molinaro, R. Short Call Poster 24

Momotani, N. Short Call Oral 5

Morel, O. Short Call Oral 6

Morris, 1. Short Call Oral 7

Mudunov, A. Short Call Poster 48

Munizza, O.N. Short Call Poster 17

Murashima, A. Short Call Oral 5

Myrie, J. Short Call Oral 8

Nassar, A. Short Call Poster 44 Nguyen, A.Q. Short Call Poster 37

Nguyen, M.T. Short Call Poster 23

Nikiforov, Y. Short Call Poster 64

Nikiforova, M. Short Call Poster 64

Nunes da Silva, T. Short Call Poster 21

Nygaard, B. Short Call Oral 4

Odzharova, A. Short Call Poster 48

Ohashi, Y. Short Call Oral 5

Oltmann, S. Short Call Poster 24

Ong, R.S. Short Call Poster 32, Short Call Poster 33

Onose, H. Short Call Oral 5

Orloff, L. Short Call Poster 23

Otto, K.J. Short Call Poster 29

Owonikoko, T.K. Short Call Oral 8

Pace, M. Short Call Oral 7

Palaniappan, L.P. Short Call Poster 23

Pan, H. Short Call Poster 11, Short Call Poster 72

Panebianco, F. Short Call Poster 64

Paschke, R. Short Call Poster 69

Patel, S. Short Call Oral 7

Patti, M. Short Call Poster 53

Payne, R. Short Call Poster 46

Pearce, E. Short Call Poster 60

Pesquero, J. Short Call Poster 59
Phelan, V.H. Short Call Oral 8

Pothuri, P. Short Call Poster 70

Pozdeyev, N. Short Call Poster 25

Preite, N.Z. Short Call Poster 53

Pretorius, H.T. Short Call Poster 12

Pribitkin, E. Short Call Poster 55

Qu, N. Short Call Poster 41

Quadri, P. Short Call Poster 62

Raef, H. Short Call Poster 47

Ragusa, F. Short Call Poster 14, Short Call Poster 15

Ralhan, R. Short Call Oral 2

Ramalingam, S.S. Short Call Oral 8

Rana, N. Short Call Poster 42

Randle, R. Short Call Poster 68

Ribeiro, M.O. Short Call Poster 53

Rivas Mejia, A.M. Short Call Poster 44

Rix, U. Short Call Poster 19

Robbins, R.J. Short Call Poster 37

Roman, B. Short Call Oral 7

Romanov, I. Short Call Poster 48

Rosenthal, R. Short Call Poster 62

Ruschenburg, I. Short Call Poster 69

Rusu, D. Short Call Oral 6

Saba, N.F. Short Call Oral 8

Sabra, M. Short Call Oral 7, Short Call

Poster 40

Sachmechi, I. Short Call Poster 70

Salehian, B. Short Call Poster 71

Schlumberger, M.J. Short Call Oral 6,

Short Call Poster 42

Schnitzer Perlman, T. Short Call Poster 52

Schroeder, E. Short Call Poster 22

Schvartz, C. Short Call Oral 6

Schweppe, R. Short Call Poster 19

Sewell, B. Short Call Poster 52

Shah, B. Short Call Poster 10

Shah, J. Short Call Oral 7

Shaha, A. Short Call Oral 7

Shakirova, G. Short Call Poster 16

Sharma, V. Short Call Poster 25,

Short Call Poster 33

Shawa, H. Short Call Poster 32

Shi, R. Short Call Poster 41

Shin, D. Short Call Oral 8

Shobab', L. Short Call Poster 36

Sidhu, S.B. Short Call Poster 35

Sikder, S. Short Call Poster 54

Singh, S. Short Call Poster 70

Sippel, R.S. Short Call Poster 68

Skaff, F. Short Call Poster 47

Smallridge, R.C. Short Call Poster 44

Smith, A. Short Call Poster 52

Song-Yang, J. Short Call Poster 50, Short Call Poster 51

Soto-Becerra, P. Short Call Poster 58

Srivatsa, S. Short Call Oral 8

Stagnaro-Green, A. Short Call Poster 60

Steffensen, R. Short Call Oral 4

Steuer, C.E. Short Call Oral 8

Stinson, R.M. Short Call Oral 8 
Sugitani, I. Short Call Poster 42

Superti-Furga, G. Short Call Poster 19

Tajiri, J. Short Call Oral 5

Talukder, A. Short Call Poster 45

Tan, A. Short Call Poster 19

Tarasova, V.D. Short Call Poster 40

Tasciotti, E. Short Call Poster 24

Taylor, S. Short Call Poster 51

Telatar, M. Short Call Poster 71

Teytelboym, O. Short Call Poster 54

Thiesen, A. Short Call Oral 1

Tighe, L. Short Call Poster 13

Timmaraju, V. Short Call Poster 50, Short Call Poster 51

Toofan, M. Short Call Poster 12

Toraldo, G. Short Call Poster 30, Short Call Poster 43

Toubert, M. Short Call Oral 6

Tuluc, M. Short Call Poster 55

Tuttle, R.M. Short Call Oral 7, Short Call Poster 40

Udovcic, M. Short Call Poster 38 Ullmann, M. Short Call Poster 69 Untch, B. Short Call Oral 7
Valderrabano, P. Short Call Poster 29

Vance, K. Short Call Poster 16

Varella Lima Teixeira, P. Short Call Poster 59

Vera, P. Short Call Oral 6

Villarreal, R.I. Short Call Poster 25

Voigt, R.Z. Short Call Poster 53

Vos, L. Short Call Oral 1

Walfish, P.G. Short Call Oral 2

Walsh, N.J. Short Call Poster 45

Wang, C. Short Call Poster 65, Short Call Poster 66

Wang, L. Short Call Poster 11

Wang, P. Short Call Oral 1

Wang, Q. Short Call Poster 11

Wang, Y. Short Call Poster 72

Wang, Z. Short Call Poster 42

Weiss, R.E. Short Call Poster 42

Wendt, E. Short Call Poster 68

Wexler, J.A. Short Call Poster 35, Short Call Poster 56

Whiting, A. Short Call Oral 7

Whitworth, S. Short Call Poster 22

Williams, D. Short Call Oral 1

Williams, M. Short Call Oral 3

Willman, J. Short Call Poster 52
Wirth, L.J. Short Call Poster 42

Wong, A.W. Short Call Poster 60

Wong, R. Short Call Oral 7

Wu, Q. Short Call Poster 72

Wu, Y. Short Call Poster 42

Xu, J. Short Call Poster 72

Yan, C. Short Call Poster 11

Yang, H. Short Call Poster 11

Yang, X. Short Call Poster 72

Yoshikawa, H. Short Call Oral 5

Zelada, H. Short Call Poster 58

Zerdoud, S. Short Call Oral 6

Zhang, B. Short Call Poster 72

Zhang, C. Short Call Oral 8

Zhang, M. Short Call Poster 11

Zhang, S. Short Call Poster 11

Zhang, X. Short Call Poster 26, Short Call Poster 72

Zhao, J. Short Call Poster 72

Zhao, R. Short Call Poster 72

Zhou, X. Short Call Poster 11

Zhu, H. Short Call Poster 11

Zhu, S. Short Call Poster 72

Zhu, Y. Short Call Poster 27

Zozobrado, K. Short Call Poster 34 